\title{
Optical bistability and hysteresis of a hybrid metal-semiconductor nanodimer
}

\author{
A. V. Malyshev ${ }^{1,2,3, *}$ and V. A. Malyshev ${ }^{3,4}$ \\ ${ }^{1}$ GISC, Departamento de Física de Materiales, Universidad Complutense, E-28040 Madrid, Spain \\ ${ }^{2}$ Ioffe Physical-Technical Institute, 26 Politechnicheskaya str., 194021 St. Petersburg, Russia \\ ${ }^{3}$ Centre for Theoretical Physics and Zernike Institute for Advanced Materials, University of Groningen, Nijenborgh 4 , \\ NL-9747 AG Groningen, The Netherlands \\ ${ }^{4}$ St. Petersburg State University, 198504 St. Petersburg, Russia \\ (Received 3 February 2011; revised manuscript received 19 April 2011; published 26 July 2011)
}

\begin{abstract}
Optical response of an artificial composite nanodimer comprising a semiconductor quantum dot and a metal nanosphere is analyzed theoretically. We show that internal degrees of freedom of the system can manifest bistability and optical hysteresis as functions of the incident field intensity. We argue that these effects can be observed for real-world systems, such as a CdSe quantum dot and an Au nanoparticle hybrid. These properties can be revealed by measuring the optical hysteresis of Rayleigh scattering. We also show that the total dipole moment of the system can be switched abruptly between its two stable states by small changes in the excitation intensity. The latter promises various applications in the field of all-optical processing at the nanoscale, the most basic of them being the volatile optical memory.
\end{abstract}

DOI: 10.1103/PhysRevB.84.035314

PACS number(s): 78.67.-n

\section{INTRODUCTION}

Arrays of metallic nanoparticles (MNPs; often referred to as plasmonic arrays) are widely recognized as potential building blocks for nanoscale optical circuitry ${ }^{1-9}$ (see also Ref. 10 for an overview). Recently, a number of groups have reported fascinating properties of artificial molecules comprised of a semiconductor quantum dot (SQD) in the proximity of an MNP. ${ }^{11-19}$ Nonlinear Fano resonances ${ }^{12,13}$ and bistability in the absorption spectrum, ${ }^{13,14}$ control of exciton emission of the SQD (inhibition or enhancement), ${ }^{15}$ and variable quenching of SQD photoluminescence by proximate gold nanoparticles, ${ }^{11}$ as well as "metamolecular" resonances ${ }^{16}$ inhibition of optical excitation and enhancement of Rabi flopping, ${ }^{17}$ tunable nanoswitching, ${ }^{18}$ and gain without inversion, ${ }^{19}$ have been predicted. The role of the multipole SQD-MNP interaction in explaining the spectra of hybrid systems has been discussed in detail in Ref. 20. All these effects depend on both geometrical parameters and material properties of hybrid clusters, providing an excellent opportunity for more fine-grained control of spectral and dynamical properties of nanoscale objects.

We consider the simplest hybrid nanocluster, comprising an SQD and a spherical MNP-artificial hybrid diatomic nanomolecule. When this system is excited optically, the dipole moment of the optical transition in the SQD generates an additional electric field at the MNP, which is superposed on the external field. Similarly, the induced dipole moment of the MNP generates an additional electric field in the SQD. Thus, the presence of the MNP leads to a self-action (feedback) of the SQD. Together with nonlinearity of the SQD itself, this can give rise to a variety of new optical properties. In particular, if the coupling between two nanoparticles is strong enough, the self-action can result in optical bistability of the response. Note that a dimer comprised of strongly coupled two-level molecules cannot manifest bistability. ${ }^{21}$ Thus, a SQD-MNP heterodimer is a fascinating nanoscopic system exhibiting this feature.

To demonstrate the feasibility of the bistable optical response of hybrid composites, we consider a closely spaced
$\mathrm{CdSe}$ (or $\mathrm{CdSe} / \mathrm{ZnSe}$ ) SQD and an Au nanosphere. We show that for a range of geometrical parameters of the system (SQD and MNP radii and center-to-center distance), optical bistability and hysteresis can be observed in it. We also argue that because of the axial symmetry of such an artificial diatomic molecule, its state can be switched not only by traditional change of the driving field amplitude, but also by change of the incoming field polarization with respect to the molecule axis, which offers an additional mechanism of control. The fact that both the SQD and the MNP can sustain high electric fields suggests such possible applications of artificial molecules as all-optical switches and optical memory cells at the nanoscale in the visible; the two stable states of the systems have different total dipole moments, providing the possibility to store information with this degree of freedom.

The paper is organized as follows. In the next section the model and formalism are described. In Sec. III we present the standard steady-state analysis of the bistable optical response, which gives rise to the optical hysteresis addressed in Sec. IV. We discuss possible applications of the predicted effects in Sec. V, while Sec. VI summarizes the paper.

\section{FORMALISM}

We assume that the SQD-MNP hybrid molecule is embedded in a dielectric host with permeability $\varepsilon_{b}$ and is driven by a linearly polarized external electric field with amplitude $\mathbf{E}_{0}$ and frequency $\omega$. Figure 1 shows the schematics of the system. The SQD is modeled as a two-level system with transition frequency $\omega_{0}$ and optical transition dipole moment $\boldsymbol{\mu}$. It is treated quantum mechanically within the framework of the Maxwell-Bloch equations for the $2 \times 2$ density matrix $\rho_{m n}(m, n=0,1)$. The MNP is considered classically; the response of the MNP is described by its frequency-dependent scalar polarizability within the point dipole approximation (this can easily be generalized for the case of more complex shapes of the MNP by considering an appropriate polarizability tensor). All sizes of the system (the SQD and MNP radii and the SQD-MNP center-to-center 


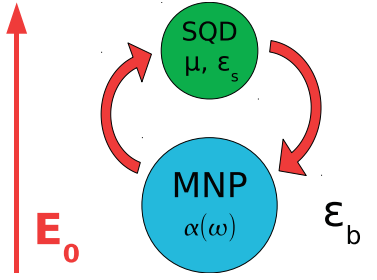

FIG. 1. (Color online) Schematics of the hybrid SQD-MNP system embedded in a homogeneous dielectric background with permeability $\varepsilon_{b}$ and subjected to an external field with amplitude $\mathbf{E}_{0}$. The SQD optical transition dipole moment and the semiconductor dielectric constant are denoted $\boldsymbol{\mu}$ and $\varepsilon_{s}$, respectively, while $\alpha(\omega)$ is the MNP polarizability. The curved arrows symbolize the dipole-dipole SQD-MNP interaction.

distance) are assumed to be small enough to neglect retardation effects and to consider both particles as point dipoles. The rotating-wave approximation is used throughout this paper, so that all time-dependent quantities represent amplitudes of the corresponding characteristics of the SQD, the set of equations for which reads

$$
\begin{gathered}
\dot{Z}=-\gamma(Z+1)-\frac{1}{2}\left[\Omega R^{*}+\Omega^{*} R\right], \\
\dot{R}=-(i \Delta+\Gamma) R+\Omega Z,
\end{gathered}
$$

where $Z=\rho_{11}-\rho_{00}$ is the population difference between the SQD excited and the SQD ground states, $R$ is the amplitude of the off-diagonal density matrix element defined through $\rho_{10}=-(i / 2) R \exp (-i \omega t), \gamma$ and $\Gamma$ are the relaxation constant of the population and the dipole dephasing, respectively, $\Delta=$ $\omega_{0}-\omega$ is the detuning of the driving field from the SQD resonance, and $\Omega=\mu \mathbf{E} / \hbar$ is the electric field (in frequency units) acting inside the SQD. The field acting on the SQD is equal to the sum of the external field $\mathbf{E}_{0}$ and the field produced by the induced dipole moment $\mathbf{P}_{\mathrm{MNP}}$ of the MNP. The field $\mathbf{E}$ inside the SQD is reduced by the factor $\varepsilon_{s}^{\prime}=\left(\varepsilon_{s}+2 \varepsilon_{b}\right) /\left(3 \varepsilon_{b}\right)$, where $\varepsilon_{s}$ is the permeability of the SQD (see, e.g., Ref. 22 or Ref. 23, chap. V, p. 138):

$$
\mathbf{E}=\frac{1}{\varepsilon_{\mathrm{s}}^{\prime}}\left(\mathbf{E}_{0}+\frac{\widehat{\mathbf{S}} \mathbf{P}_{\mathrm{MNP}}}{\varepsilon_{b} d^{3}}\right) .
$$

Here, $\widehat{\mathbf{S}}=\operatorname{diag}(-1,-1,2)$ is the angular part of the dipole field Green's tensor (the $z$ axis being parallel to the system axis), $d$ is the SQD-MNP center-to-center distance, and $\mathbf{P}_{\mathrm{MNP}}$ is given by

$$
\mathbf{P}_{\mathrm{MNP}}=\varepsilon_{b} \alpha(\omega)\left(\mathbf{E}_{0}+\frac{\widehat{\mathbf{S}} \mathbf{P}_{\mathrm{SQD}}}{\varepsilon_{b} d^{3}}\right),
$$

where $\alpha(\omega)=a^{3} \gamma(\omega)$ is the classical frequency-dependent polarizability of the MNP, $a$ being its radius, $\gamma(\omega)=\left[\varepsilon_{M}(\omega)-\right.$ $\left.\varepsilon_{b}\right] /\left[\varepsilon_{M}(\omega)+2 \varepsilon_{b}\right]$, and $\varepsilon_{M}(\omega)$ is the dielectric function of the metal. We do not take into account the corrections to the polarizability due to the depolarization shift and radiative damping, ${ }^{24}$ which are negligible for nanoparticle sizes of our interest $(\leqslant 10 \mathrm{~nm})$. The second term in parentheses in Eq. (3) is the field produced by the SQD dipole moment $\mathbf{P}_{\mathrm{SQD}}=-i \boldsymbol{\mu} R$ at the MNP.

The exciton radius in CdSe is about $5 \mathrm{~nm},{ }^{25}$ while the typical radius of the considered SQD is about $1.5-2 \mathrm{~nm}$, so the wave functions involved in the optical transition are extended over the whole dot. In deriving Eq. (3) we therefore used the approximation of the homogeneous electric polarization of the whole SQD volume. In this case the dipole field around the SQD is screened by the bare background dielectric constant only. ${ }^{22}$ Note that the dipole moment $\mathbf{P}_{\mathrm{SQD}}$ is calculated quantum mechanically and accounts for the screening which results from the SQD dielectric response (see below). Finally, for the total electric field inside the SQD we obtain

$$
\mathbf{E}=\frac{1}{\varepsilon_{\mathrm{s}}^{\prime}}\left[\mathbf{1}+\frac{\gamma(\omega) a^{3}}{d^{3}} \widehat{\mathbf{S}}\right] \mathbf{E}_{0}+\frac{\gamma(\omega) a^{3}}{\varepsilon_{b} \varepsilon_{s}^{\prime} d^{6}} \widehat{\mathbf{S}}^{2} \mathbf{P}_{\mathrm{SQD}} .
$$

As shown by Eq. (4), the presence of the MNP results in two effects: the first term accounts for the renormalization of the external field amplitude $\mathbf{E}_{0}$, while the second represents the self-action of the SQD via the MNP; the field inside the SQD depends on the dipole moment of the SQD itself.

The effect of the self-action on the dynamics of the SQDMNP hybrid nanomolecule can be revealed after substituting Eq. (4) into Eq. (1b) and representing $\Omega$ in the form

$$
\Omega=\widetilde{\Omega}_{0}-i G R,
$$

with $\widetilde{\Omega}_{0}$ and $G$ given by

$$
\begin{gathered}
\widetilde{\Omega}_{0}=\frac{1}{\varepsilon_{s}^{\prime}}\left[1+\frac{a^{3} \gamma(\omega)}{d^{3}} \frac{\boldsymbol{\mu} \widehat{\mathbf{S E}}_{0}}{\hbar \Omega_{0}}\right] \Omega_{0}, \\
G=\frac{\gamma(\omega) a^{3}}{\varepsilon_{b} \varepsilon_{s}^{\prime} \hbar d^{6}} \widehat{\boldsymbol{S}}^{2} \boldsymbol{\mu},
\end{gathered}
$$

where $\Omega_{0}=\mu \mathbf{E}_{0} / \hbar$ is the Rabi frequency of the bare external field, $\widetilde{\Omega}_{0}$ is the renormalized Rabi frequency, and $G$ is the feedback parameter. The latter absorbs all information governing the SQD self-action, such as the material constants, geometry of the system, and details of the interaction (e.g., contributions of higher multipoles). ${ }^{20}$

In a number of recent publications dealing with the same system, a different formula for the constant $G$ was used in which the factor $\varepsilon_{\mathrm{s}}^{\prime}$ appears squared in the denominator of the $G .^{12-20}$ The second factor, $\varepsilon_{\mathrm{s}}^{\prime}$, is supposed to take into account the screening of the dipole field by the SQD dielectric response. We note, however, that it is the product $G R$ that determines the latter field, and as shown by Eq. (8b), $R \propto \widetilde{\Omega}_{0} \propto \Omega_{0} / \varepsilon_{\mathrm{s}}^{\prime}$, so the dipole field $G R$ is already additionally screened.

The feedback $G$ is the most important parameter of the theory; once it is calculated it determines the nonlinear properties of the SQD response. Using Eq. (5), Eq. (1b) can be rewritten in the form

$$
\dot{R}=-\left[\left(\Gamma-G_{\mathrm{I}} Z\right)+i\left(\Delta+G_{\mathrm{R}} Z\right)\right] R+\widetilde{\Omega}_{0} Z,
$$

with $G_{\mathrm{R}}=\operatorname{Re}(G)$ and $G_{\mathrm{I}}=\operatorname{Im}(G)$. From Eq. (7) two consequences of the SQD self-action become apparent: (i) the renormalization of the SQD resonance frequency $\omega_{0} \mapsto \omega_{0}+$ $G_{\mathrm{R}} Z$ and (ii) the renormalization of the dipole dephasing rate $\Gamma \mapsto \Gamma-G_{\mathrm{I}} Z$. Both renormalized quantities depend on the population difference $Z$. Similar renormalizations originate from the local field correction in the nonlinear optical response of dense gaseous assemblies of two-level systems, ${ }^{26}$ optically dense thin films, ${ }^{27}$ and linear molecular aggregates. ${ }^{28}$ The population dependencies of the SQD resonance frequency 


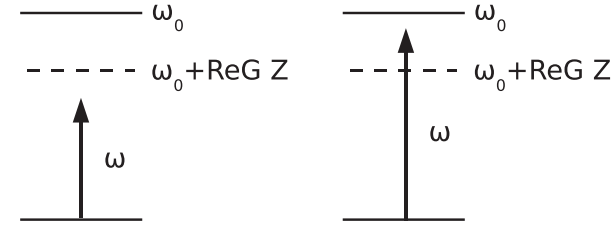

FIG. 2. Two possible types of SQD excitation. Left: The excitation frequency $\omega$ lies below the renormalized SQD transition frequency $\omega_{0}-G_{\mathrm{R}}\left(\Delta>G_{\mathrm{R}}\right)$; as $Z$ increases with the excitation, the system is driven farther out of resonance. Right: $\omega>\omega_{0}-G_{\mathrm{R}}$ $\left(\Delta<G_{\mathrm{R}}\right.$ ); the excitation drives the system into resonance, which favors the occurrence of bistability.

and the dipole dephasing rate provide a feedback mechanism, which results in a number of fascinating effects.

Let us assume that both $G_{\mathrm{R}}>0$ and $G_{\mathrm{I}}>0$, which is the case for a CdSe SQD conjugated with a golden MNP. Then the renormalized resonance frequency increases with the excitation intensity, ranging from $\omega_{0}-G_{\mathrm{R}}(Z=-1$ in the ground state) to $\omega_{0}+G_{\mathrm{R}}(Z=1$ in the excited state). In this case the response of the system depends on the relative position of the excitation frequency $\omega$ with respect to the renormalized SQD transition frequency $\omega_{0}-G_{\mathrm{R}}$. Thus, if $\omega<\omega_{0}-G_{\mathrm{R}}$ or, equivalently, $\Delta>G_{\mathrm{R}}$ (see Fig. 2, left) then the excitation is driving the SQD out of resonance, so that the SQD is becoming less absorptive. On the contrary, if $\omega>\omega_{0}-G_{\mathrm{R}}$ or $\Delta<G_{\mathrm{R}}$ (see Fig. 2, right), the SQD is being driven into self-sustaining resonance by the incoming field. This is the case of positive loopback. In this case, apart from the usual linear "weak-field" solution, the second kind of stable state can occur, which results from the above-mentioned positive feedback mechanism. We show below that the latter has a threshold character, giving rise to bistability and hysteresis of the system response characteristics.

\section{STEADY-STATE ANALYSIS}

First, we analyze Eqs. (1a) and (1b) under steady-state conditions $(\dot{Z}=\dot{R}=0)$ to obtain stationary states of the system. The corresponding solutions read

$$
\begin{gathered}
\frac{\left|\widetilde{\Omega}_{0}\right|^{2}}{\gamma \Gamma}=-\frac{Z+1}{Z} \frac{\left|\left(\Gamma-G_{\mathrm{I}} Z\right)+i\left(\Delta+G_{\mathrm{R}} Z\right)\right|^{2}}{\Gamma^{2}}, \\
R=\frac{Z \widetilde{\Omega}_{0}}{\left(\Gamma-G_{\mathrm{I}} Z\right)+i\left(\Delta+G_{\mathrm{R}} Z\right)} .
\end{gathered}
$$

Equation (8a) is of the third order in $Z$ and therefore may have three real solutions, depending on the values of $\Delta, \Gamma$, $G_{\mathrm{R}}$, and $G_{\mathrm{I}}$. The same applies to the SQD dipole moment amplitude $R$.

Hereafter, we consider a CdSe SQD in the vicinity of an $\mathrm{Au}$ MNP and use the following set of parameters: the transition energy $\hbar \omega_{0}=2.36 \mathrm{eV}$ (which corresponds to the optical transition in a 3.3-nm SQD), the SQD dielectric constant $\varepsilon_{s}=6.2$, the SQD transition dipole moment $\mu=0.65 \mathrm{e} \cdot \mathrm{nm},{ }^{12}$ the MNP radius $a=10 \mathrm{~nm}$, the SQD-MNP center-to-center distance $d=17 \mathrm{~nm}$, the host dielectric constant $\varepsilon_{b}=1$, and the relaxation constants $\gamma$ and $\Gamma$ are defined through $1 / \gamma=$
$0.8 \mathrm{~ns}$ and $1 / \Gamma=0.3 \mathrm{~ns}^{13}$ To calculate the polarizability $\gamma(\omega)$ of the MNP, we used the tabulated data for the permittivity of gold from Ref. 29. For these parameters $G=G_{\mathrm{R}}+i G_{\mathrm{I}}=$ $(25.4+10.6 i) \Gamma$. Note that the frequency domain of our interest is a narrow region in the vicinity of the SQD resonance, with a width of about several units of $\Gamma$ (see below), which is much smaller than the width of the MNP plasmonic resonance. We therefore neglected the frequency dependence of the MNP polarizability when calculating the feedback parameter $G$ and used $\gamma(\omega) \approx \gamma\left(\omega_{0}\right)$.

Figure 3 shows the solution of Eqs. (8a) and (8b) for the set of parameters specified above and different detunings $\Delta$. As shown in the plots, within a window of $-3.3 \Gamma \leqslant \Delta \leqslant 13.8 \Gamma$, the field dependence of $Z$ and $R$ have three allowed values for a given intensity $\left|\Omega_{0}\right|^{2} /(\gamma \Gamma)$. The upper limit of the window, $\Delta=13.8 \Gamma$, corresponds to the excitation frequency $\omega=\omega_{0}-13.8 \Gamma$, which lies above the renormalized SQD resonance frequency $\omega_{0}-G_{\mathrm{R}}=\omega_{0}-25.4 \Gamma$ (the positive loopback case). The lower limit of the window, $\Delta=-3.3 \Gamma$, is negative and the corresponding frequency lies above the bare resonance. Nevertheless, the SQD can still be driven into self-sustaining resonance by the external field. At larger $\omega$ (larger negative $\Delta$ ), resonance between the excitation and the SQD cannot be attained because it requires a significant positive population difference $Z$ that is unreachable under stationary conditions: due to the saturation effect, the upper limit for the population difference is $Z=0$ in the steady state. Because of this, the bistability effect disappears for large negative detunings.
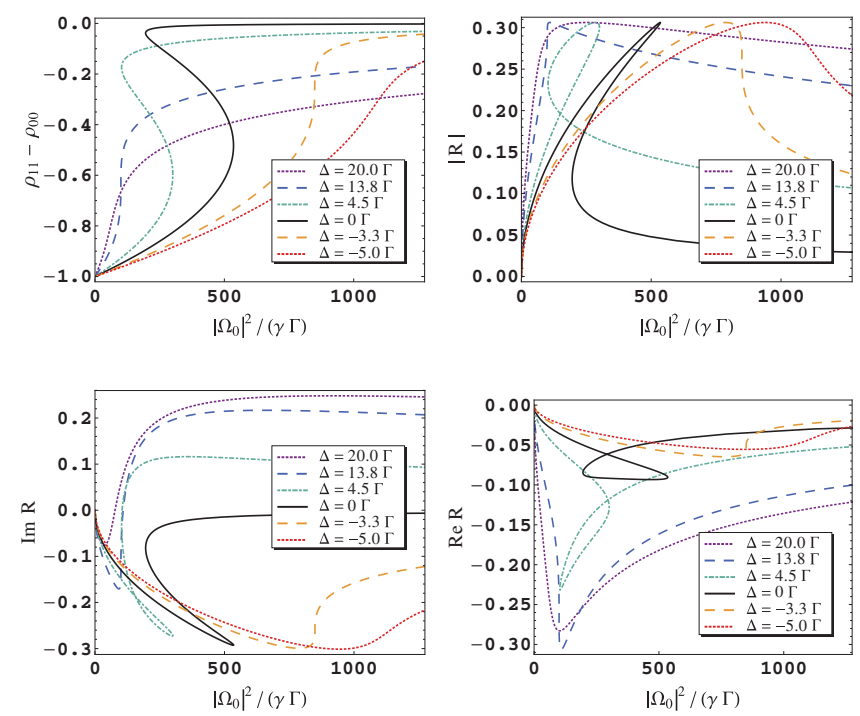

FIG. 3. (Color online) Stationary solutions to Eqs. (8a) and (8b) for a hybrid nanomolecule comprising a 3.3-nm CdSe SQD and a $20-\mathrm{nm}$ Au sphere separated by a center-to-center distance of $17 \mathrm{~nm}$ (other parameters are described in the text). Solutions are calculated for different detuning $\Delta$ as functions of the normalized external field intensity $\Omega_{0} / \sqrt{\gamma \Gamma}$. The upper two panels show the population difference $Z=\rho_{11}-\rho_{00}$ (left) and the SQD dipole moment amplitude $|R|$ (right). The lower-left panel displays the absorptive part of the dipole amplitude, $\operatorname{Im}(R)$; the lower-right panel, its dispersive part, $\operatorname{Re}(R)$. 
Optical bistability can therefore be observed within a window of detunings in the vicinity of the SQD transition frequency. The width of this window is typically of the order of several units or tens of $\Gamma$. In small SQDs the dipole dephasing time is usually strongly temperature dependent and can change from nanoseconds at low temperatures to less than a picosecond at room temperature. ${ }^{30}$ The bistability exists only if the feedback parameter $G$ exceeds some threshold value. If $G_{\mathrm{I}}=0$, the condition for bistability to occur is $G_{\mathrm{R}} \geqslant 4 \Gamma,{ }^{26}$ while if $G_{\mathrm{R}}=0$, the condition is $G_{\mathrm{I}} \geqslant 8 \Gamma{ }^{31}$ The value of $G$ is determined by the geometry and material properties and cannot be increased arbitrarily. Therefore, these criteria can be used to choose suitable materials and system configuration.

We note that only the population difference $Z$ manifests the standard S-shaped curves, while all the quantities related to the SQD dipole amplitude $R$ exhibit more exotic coiled curves. As we show below, the latter leads to completely different types of hysteresis loops for these quantities.

\section{OPTICAL HYSTERESIS}

We performed time-domain calculations with the external field intensity $\left|\Omega_{0}\right|^{2} /(\gamma \Gamma)$ being adiabatically swept back and forth across the bistability region (sweeping speeds are given in figure captions), monitoring the evolution of the system to determine which steady-state branches are stable. The results are shown in Fig. 4. For the population difference $Z$ and the absorptive part of the SQD dipole moment $\operatorname{Im}(R)$, we find the standard behavior. Upon increasing the applied intensity, the system follows the lower (stable) branch until the intensity reaches the critical value at which the system switches to the upper branch (which is also stable). Upon sweeping the intensity back, the system stays on the upper branch and then switches back down to the lower one at the other critical
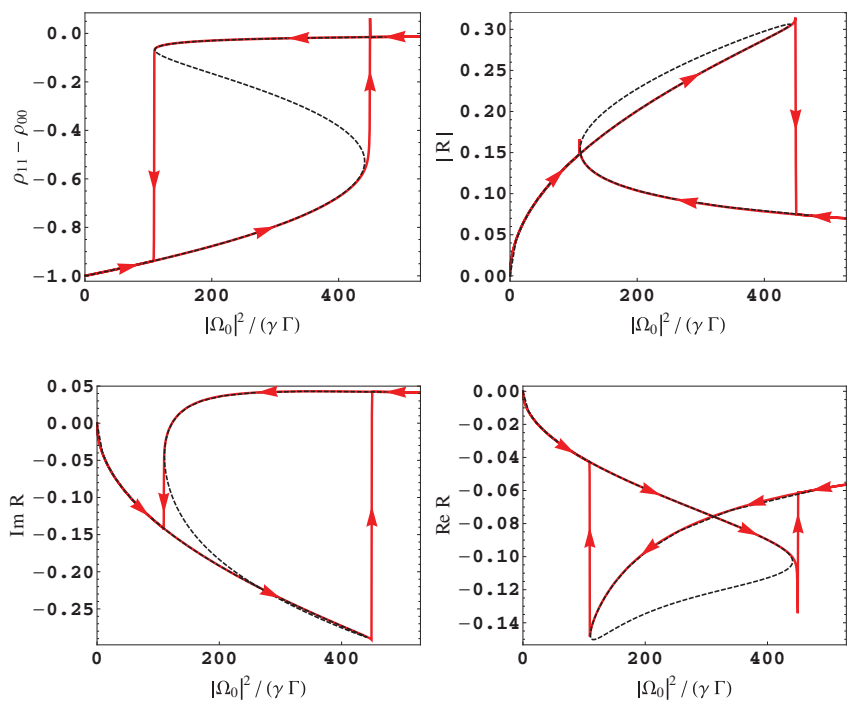

FIG. 4. (Color online) Optical hysteresis loops of the population difference $Z=\rho_{11}-\rho_{00}$ and the SQD dipole moment amplitude $R$ obtained by solving Eqs. (1a) and (1b) with the external field intensity $\left|\Omega_{0}\right|^{2} / \sqrt{\gamma \Gamma}$ being adiabatically swept back and forth across the bistability region (the sweeping speed of the renormalized Rabi frequency $\widetilde{\Omega}_{0} / \sqrt{\gamma \Gamma}$ is $2.1 \times 10^{-3} \Gamma$ ) The detuning from the resonance is $\Delta=1.5 \Gamma$. All other parameters are as in Fig. 3 . intensity, completing the hysteresis loop. The intermediate branch cannot be revealed by the adiabatic sweeping of the field because it is unstable, which can also be checked by the standard stability analysis.

Both $|R|$ and $\operatorname{Im}(R)$ behave in a very different manner, manifesting hysteresis loops with kinks. In the case of $|R|$ the upper branch is unstable and the hysteresis loop is triangular, while $\operatorname{Im}(R)$, with its unstable lower branch, has an even more complicated bow-tie hysteresis curve. To the best of our knowledge, no such optical hysteresis loops have been either predicted or observed so far.

These unusual properties are also expected to manifest themselves in the MNP dipole moment because it depends on that of the SQD [see Eq. (3)] and can therefore be switched abruptly as well.

\section{RAYLEIGH SCATTERING AND OPTICAL STORAGE}

In experiments, the intensity of the Rayleigh scattering can be measured. The amplitude of this characteristic is known to be proportional to the squared absolute value of the total system dipole moment $\left|P_{\mathrm{SQD}}+P_{\mathrm{MNP}}\right|^{2}$ and is therefore expected to manifest bistability as well. We plot the amplitude of the Rayleigh scattering in Fig. 5 for different values of detuning $\Delta$. The figure shows that various types of hysteresis curves can be observed: the standard loop (for $\Delta=0$ ) as well as more exotic triangular loops $(\Delta=1.5 \Gamma$ and $\Delta=8 \Gamma)$ and the bow-tie one $(\Delta=4.5 \Gamma)$. The latter two types of hysteresis curves are characteristic for the SQD dipole moment (see Fig. 4), which suggests that the major contribution to the scattering is coming from the SQD. To confirm the latter we calculated the relative contribution of the SQD dipole moment to the total scattering
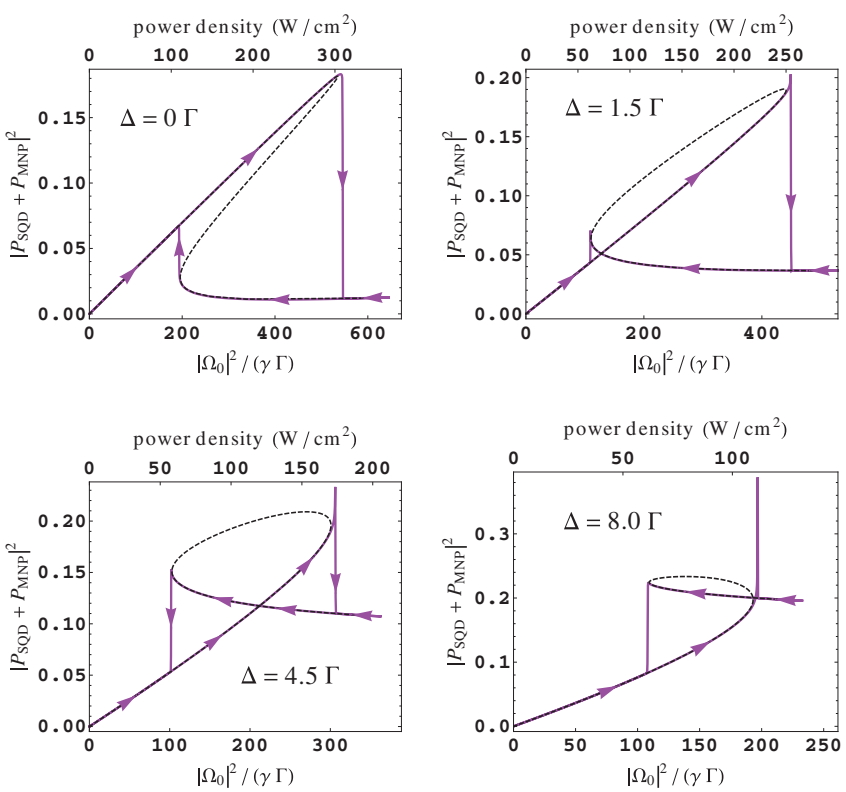

FIG. 5. (Color online) Rayleigh scattering intensity $\mid P_{\mathrm{SQD}}+$ $\left.P_{\mathrm{MNP}}\right|^{2}$ in units of $|\mu|^{2}$ for different values of $\Delta$ (indicated in the figures) as a function of excitation intensity. The corresponding power density is shown on the upper axis. Sweeping speeds of the renormalized Rabi frequency $\widetilde{\Omega}_{0} / \sqrt{\gamma \Gamma}$ in units of $\Gamma$ are of the order of $10^{-3}$ for all values of $\Delta$. All other parameters are as in Fig. 3 . 
amplitude, $\left|P_{\mathrm{SQD}}\right|^{2} /\left|P_{\mathrm{SQD}}+P_{\mathrm{MNP}}\right|^{2}$, which turned out to be of the order of unity across the whole hysteresis region. Within this region the contribution of the MNP to the scattered field is typically an order of magnitude less than that of the SQD for the chosen set of parameters.

It is to be noted that the ratio of the scattering intensity in the two stable states can be as high as about 25 for $\Delta=0$. Such a high contrast suggests that the system can be used as an optical memory cell: the lower intensity can represent a logical 0 ; the higher intensity, a logical 1 . The state of the cell can be switched by sweeping the excitation power across the bistability region. Another possibility for switching the cell is to maintain the power constant while changing the incident-field polarization. The latter mechanism is characteristic for this particular system due to its axial symmetry. We point out that this memory is a volatile one, as it requires constant pumping.

\section{SUMMARY}

We have investigated theoretically the optical response of a hybrid "artificial molecule" comprised of a closely spaced spherical SQD (modeled as a two-level system) and a metal nanosphere (considered classically), which are coupled by the dipole-dipole interaction. The interaction results in a self-action of the SQD via the MNP, leading to population dependence of the SQD transition frequency and relaxation constant of the SQD dipole moment. This provides a feedback mechanism resulting in several fascinating effects. Thus, we found that the system can manifest bistability and optical hysteresis. In particular, the total dipole moment of the system can be switched between its two stable states by the incoming field.
The latter suggests such possible applications as optical memory cells and all-optical switches at the nanoscale in the visible.

Because the SQD-MNP dipole-dipole interaction depends on the orientation of the dipole moments of the two particles, switching can be achieved not only by the traditional control by the incident amplitude, but also by change of the polarization of the incoming field with respect to the system axis. Our calculations performed for typical system parameters, such as those of $\mathrm{CdSe}$ or $\mathrm{CdSe} / \mathrm{ZnSe}$ quantum dot and Au nanoparticle complexes, predict the optical bistability of an SQD-MNP artificial molecule. Modern methods of single-particle spectroscopy ${ }^{30,32-34}$ could probably be used to discover the predicted effects experimentally.

To conclude, we have considered the simplest diatomic hybrid artificial nanomolecule. We expect, however, that more complicated clusters (such as an SQD surrounded by several MNPs, as considered in Ref. 15) can also exhibit these effects because in such systems nanoparticles are just playing the role of a "resonator" and provide feedback to the nonlinear two-level system. Anisotropy of nanoparticles can also easily be accounted for by using an appropriate tensor instead of the scalar polarizability. Finally, we note that a very interesting aspect of this kind of system is the direction of the total induced dipole moment, which can also be bistable.

\section{ACKNOWLEDGMENTS}

A.V.M. acknowledges support from projects MOSAICO (FIS2006-01485) and BSCH-UCM (PR58/08) and the Ramón y Cajal program (Ministerio de Ciencia e Innovación de España) and is grateful to the University of Groningen for hospitality.
*Corresponding author: a.malyshev@ fis.ucm.es

${ }^{1}$ M. Quinten, A. Leitner, R. M. Krenn, and F. R. Aussenegg, Opt. Lett. 23, 1331 (1998).

${ }^{2}$ M. L. Brongersma, J. W. Hartman, and H. A. Atwater, Phys. Rev. B 62, R16356 (2000).

${ }^{3}$ K. Li, M. I. Stockman, and D. J. Bergman, Phys. Rev. Lett. 91, 227402 (2003).

${ }^{4}$ M. I. Stockman, Phys. Rev. Lett. 93, 137404 (2004).

${ }^{5}$ D. S. Citrin, Nano Lett. 4, 1561 (2004).

${ }^{6}$ J. V. Hernández, L. D. Noordan, and F. J. Robicheaux, J. Phys. Chem. B 109, 15808 (2005).

${ }^{7}$ R. René de Waele, A. F. Koenderink, and A. Polman, Nano Lett. 7, 2004 (2007).

${ }^{8}$ A. V. Malyshev, V. A. Malyshev, and J. Knoester, Nano Lett. 8, 2369 (2008).

${ }^{9}$ M. J. Zheng, J. J. Xiao, and K. W. Yu, J. Appl. Phys. 106, 113307 (2009).

${ }^{10}$ S. A. Maier, Plasmonics: Fundamentals and Applications (Springer, New York, 2007).

${ }^{11}$ T. Pons, I. L. Medintz, K. E. Sapsford, S. Higashiya, A. F. Grimes, D. S. English, and H. Mattoussi, Nano Lett. 7, 3157 (2007).

${ }^{12}$ W. Zhang, A. O. Govorov, and G. W. Bryant, Phys. Rev. Lett. 97, 146804 (2006).

${ }^{13}$ R. D. Artuso and G. V. Bryant, Nano Lett. 8, 2106 (2008).
${ }^{14}$ R. D. Artuso and G. W. Bryant, Phys. Rev. B 82, 195419 (2010).

${ }^{15}$ A. O. Govorov, G. W. Bryant, W. Zhang, T. Skeini, J. Lee, N. A. Kotov, J. M. Slocik, and R. R. Naik, Nano Lett. 6, 984 (2006).

${ }^{16}$ S. M. Sadeghi, Phys. Rev. B 79, 233309 (2009).

${ }^{17}$ S. M. Sadeghi, Nanotechnology 20, 225401 (2009).

${ }^{18}$ S. M. Sadeghi, Nanotechnology 21, 355501 (2010).

${ }^{19}$ S. M. Sadeghi, Nanotechnology 21, 455401 (2010).

${ }^{20}$ J.-Y. Yan, W. Zhang, S. Duan, X.-G. Zhao, and A. O. Govorov, Phys. Rev. B 77, 165301 (2008).

${ }^{21}$ V. A. Malyshev, H. Glaeske, and K.-H. Feller, Phys. Rev. A 58, 1496 (1998).

${ }^{22}$ V. V. Batygin and I. N. Toptygin, Sbornik Zadach Po Elektrodinamike 2-e izd. (M.: Nauka, 1970); Problems In Electrodynamics, 2nd ed. (Academic Press, London, 1978).

${ }^{23}$ C. F. Bohren and D. R. Huffman, Absorption and Scattering of Light by Small Particles (Wiley, New York, 1983).

${ }^{24}$ M. Meier and A. Wokaun, Opt. Lett. 8, 581583 (1983).

${ }^{25}$ A. I. Ekimov et al., J. Opt. Soc. Am. B 10, 100 (1993).

${ }^{26}$ R. Friedberg, S. R. Hartmann, and J. T. Manassah, Phys. Rev. A 39, 3444 (1989).

${ }^{27}$ M. G. Benedict, V. A. Malyshev, E. D. Trifonov, and A. I. Zaitsev, Phys. Rev. A 43, 3845 (1991). 
${ }^{28}$ V. Malyshev and P. Moreno, Phys. Rev. A 53, 416 (1996).

${ }^{29}$ P. B. Johnson and R. W. Christy, Phys. Rev. B 6, 4370 (1972).

${ }^{30}$ X. Michalet, F. Pinaud, T. D. Lacoste, M. Dahan, M. P. Bruchez, A. P. Alivisatos, and S. Weiss, Single Mol. 2, 261 (2001).

${ }^{31}$ A. M. Basharov, Zh. Eksp. Teor. Fiz. 94, 12 (1988) [JETP 67, 1741 (1988)].
${ }^{32}$ L. J. E. Anderson, K. M. Mayer, R. D. Fraleigh, Y. Yang, S. Lee, and J. H. Hafner, J. Phys. Chem. C 114, 11127 (2010).

${ }^{33}$ A. Tcherniak, J. W. Ha, S. Dominguez-Medina, L. S. Slaughter, and S. Link, Nano Lett. 10, 1398 (2010).

${ }^{34}$ L. S. Slaughter, W.-S. Chang, P. Swanglap, A. Tcherniak, B. P. Khanal, E. R. Zubarev, and S. Link, J. Phys. Chem. C 114, 4934 (2010). 